\title{
Isospectral domains with mixed boundary conditions
}

\author{
Michael Levitin ${ }^{1}$, Leonid Parnovski ${ }^{2}$, Iosif Polterovich ${ }^{3}$ \\ ${ }^{1}$ Maxwell Institute, Department of Mathematics, Heriot-Watt University, Riccarton, \\ Edinburgh EH14 4AS, U.K.; e-mail M.Levitin@ma.hw.ac.uk \\ ${ }^{2}$ Department of Mathematics, University College London, Gower Street, London \\ WC1E 6BT, U.K.; e-mail leonid@maths.ucl.ac.uk \\ ${ }^{3}$ Dépt. de mathématiques et de statistique, Université de Montréal, CP 6128 succ \\ Centre-Ville, Montreal, QC H3C 3J7, Canada; e-mail iossif@dms.umontreal.ca
}

\begin{abstract}
We construct a series of examples of planar isospectral domains with mixed Dirichlet-Neumann boundary conditions. This is a modification of a classical problem proposed by M. Kac.

Keywords: Laplacian, mixed Dirichlet-Neumann problem, isospectrality.

Published in J. Phys. A: Math. Gen. 39 2073-2082 (2006). This version corrects the statement of Theorem 5.1.
\end{abstract}

AMS classification scheme numbers: 58J53, 35P05

\section{Introduction}

Let us recall the classical question of Mark Kac, "Can one hear the shape of a drum?" $\mathrm{Kac}$. He asked whether there exist two non-isometric domains on the plane such that the spectra of the (Dirichlet) Laplacian on them coincide (such domains are called isospectral). For arbitrary planar domains it was answered negatively in [GWW] using an algebraic construction of [Sun]. See also reviews and extensions [BCDS, Bro, Bus, Ch] and references therein, as well as illustrative numerics in [BetTr]. Also, it was observed in $[\mathrm{SIHu}$ ] that the constructions of [BCDS, Ch] work in the case of domains with fractal boundaries. However, to the best of our knowledge there are still no known examples of sets of more than two non-isometric isospectral domains, as well as of non-simply connected domains. Also, Kac's question still remains open for smooth (as well as for convex) domains.

In the present paper we suggest the following modification of isospectrality question for the case of mixed Dirichlet-Neumann boundary conditions (the so called Zaremba problem [Za] ) which develops the approach suggested in [JLNP]. Some related numerically constructed (though more complicated) examples can be found in [DG].

Let $\Omega_{j} \subset \mathbb{R}^{2}, j=1,2$ be two bounded domains, their piecewise smooth boundaries being decomposed as $\partial \Omega_{j}=\overline{\partial_{D} \Omega_{j} \cup \partial_{N} \Omega_{j}}$, where $\partial_{D} \Omega_{j}, \partial_{N} \Omega_{j}$ are finite unions of open 
segments of $\partial \Omega_{j}$ and $\partial_{D} \Omega_{j} \cap \partial_{N} \Omega_{j}=\emptyset$. Suppose that there are no isometries of $\mathbb{R}^{2}$ mapping $\Omega_{1}$ onto $\Omega_{2}$ in such a way that $\partial_{D} \Omega_{1}$ maps onto $\partial_{D} \Omega_{2}$. (We shall call such pairs of domains nontrivial.) Consider on each $\Omega_{j}$ a mixed boundary value problem for the Laplacian,

$$
-\Delta u=\lambda u \quad \text { in } \Omega_{j},\left.\quad u\right|_{\partial_{D} \Omega_{j}}=0, \quad \partial u /\left.\partial n\right|_{\partial_{N} \Omega_{j}}=0
$$

and denote its spectrum by $\sigma_{D N}\left(\Omega_{j}\right)$. Our aim is to study nontrivial isospectral pairs $\Omega_{1}, \Omega_{2}$ (i.e. such that $\left.\sigma_{D N}\left(\Omega_{1}\right)=\sigma_{D N}\left(\Omega_{2}\right)\right)$. We present a series of examples of such pairs and provide some necessary conditions for mixed isospectrality.

\section{Basic example}

Let $\Omega_{1}=(0,1)^{2}$ be a unit square with $\partial_{N} \Omega_{1}=\{1\} \times(0,1)$ and $\partial_{D} \Omega_{1}=\{0\} \times[0,1] \cup$ $(0,1) \times\{0,1\}$. Let $\Omega_{2}$ be an isosceles right-angled triangle $\left\{(x, y) \in \mathbb{R}^{2}: 0<x<\right.$ $\sqrt{2}, 0<y<x\}$ with $\partial_{N} \Omega_{2}=\{\sqrt{2}\} \times(0, \sqrt{2})$ and $\partial_{D} \Omega_{2}=\{0\} \times(0, \sqrt{2}) \cup\{(x, x): x \in$ $(0, \sqrt{2})\}$ (see Figure 1)

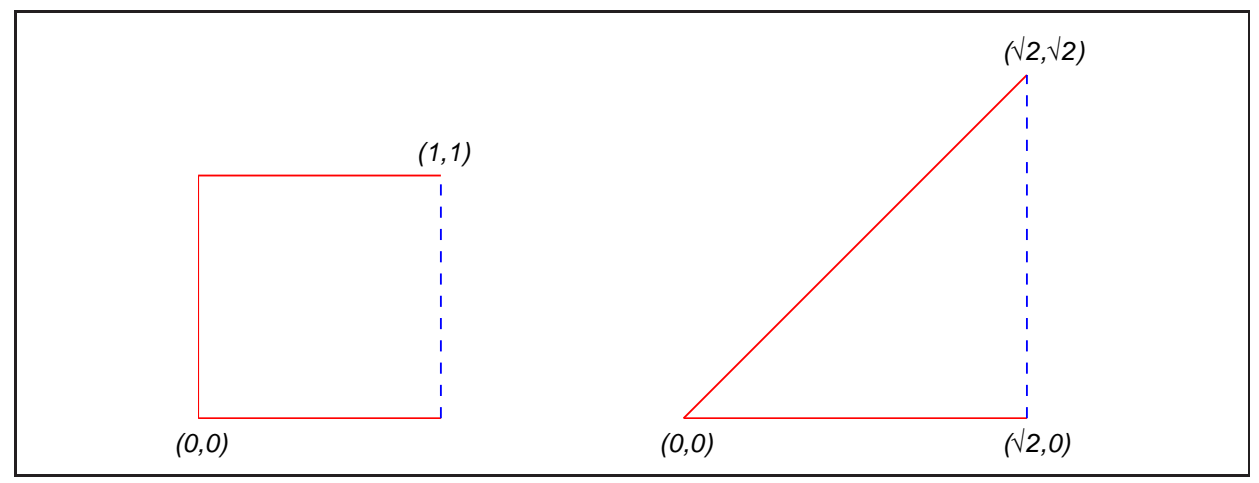

Figure 1. The unit square $\Omega_{1}$ and the isosceles triangle $\Omega_{2}$. Here and further on, red solid lines —_ denote the Dirichlet boundary conditions and blue dashed lines - - - denote the Neumann ones.

The spectra $\sigma_{D N}\left(\Omega_{j}\right)$ are easily calculated by separation of variables. The eigenfunctions for $\Omega_{1}$ are

$$
\sin ((1 / 2+m) \pi x) \sin (n \pi y), \quad \text { for } n=1,2, \ldots, m=0,1,2, \ldots,
$$

and the eigenfunctions for $\Omega_{2}$ are

$$
\begin{array}{r}
\sin \left(\frac{(1 / 2+k) \pi x}{\sqrt{2}}\right) \sin \left(\frac{(1 / 2+l) \pi y}{\sqrt{2}}\right)-\sin \left(\frac{(1 / 2+l) \pi x}{\sqrt{2}}\right) \sin \left(\frac{(1 / 2+k) \pi y}{\sqrt{2}}\right) \\
\text { for } k=0,1,2, \ldots, l=0,1,2, \ldots, k>l .
\end{array}
$$

The corresponding spectra (with account of multiplicities) are $\sigma_{D N}\left(\Omega_{1}\right)=\left\{\lambda_{m, n}\right\}$ and $\sigma_{D N}\left(\Omega_{2}\right)=\left\{\mu_{k, l}\right\}$ with

$$
\lambda_{m, n}=\frac{\pi^{2}}{4}\left((2 m+1)^{2}+4 n^{2}\right)
$$


and

$$
\mu_{k, l}=\frac{\pi^{2}}{4} \frac{(2 k+1)^{2}+(2 l+1)^{2}}{2} .
$$

Theorem 2.4. $\quad \sigma_{D N}\left(\Omega_{1}\right)=\sigma_{D N}\left(\Omega_{2}\right)$

Proof. Indeed, it is easy to check that

$$
\mu_{k, l}= \begin{cases}\lambda_{j, l+j+1} & \text { if } k-l=2 j+1, \\ \lambda_{l+j, j} & \text { if } k-l=2 j .\end{cases}
$$

On the other hand,

$$
\lambda_{m, n}= \begin{cases}\mu_{m+n, m-n} & \text { if } m \geq n, \\ \mu_{m+n, n-m-1} & \text { if } m<n .\end{cases}
$$

These two correspondences establish a bijection between $\sigma_{D N}\left(\Omega_{1}\right)$ and $\sigma_{D N}\left(\Omega_{2}\right)$.

The example above shows that isospectral domains with mixed boundary conditions can be quite simple (compared to classical Dirichlet isospectral pairs in GWW], BCDS] and numerical examples in $[\mathrm{DG}]$ ). Indeed, dependence of the spectra on boundary decomposition brings more flexibility to the problem. See also section [6 for other illustrations of this phenomenon.

We note that this example is somewhat reminiscent of Chapman's example Ch] of two disconnected Dirichlet isospectral domains: in his case the first disconnected domain is a disjoint union of a square of side one and an isosceles right triangle of side two, and the second one is a disjoint union of a rectangle with sides one and two and an isosceles right triangle of side $\sqrt{2}$.

\section{Main construction}

Example of section 2 is in fact the easiest implementation of the following algorithm for the construction of isospectral domains which we outline below.

We start by describing a suitable class of "construction blocks" which we shall later use to build pairs of planar isospectral domains. Let $a, b$ be two lines on the plane (which may be parallel), and let $K$ be a bounded open set lying in a sector formed by $a$ and $b$ (or between them if they are parallel). $K$ need not be connected, but we assume that $\partial K$ has non-empty intersections with $a$ and $b$ which we denote $\partial_{a} K$ and $\partial_{b} K$, respectively. Let $\partial_{0} K:=\partial K \backslash\left(\partial_{a} K \cup \partial_{b} K\right)$ be the remaining part of the boundary $\partial K$.

Let $T_{a}, T_{b}$ denote the reflections with respect to the lines $a$ and $b$. We first construct the domains $\Omega_{1}$ and $\Omega_{2}$ just by adding to $K$ its image under reflections $T_{a}$, $T_{b}$, respectively:

$$
\Omega_{1}:=\operatorname{Int}\left(\overline{K \cup T_{a}(K)}\right), \quad \Omega_{2}:=\operatorname{Int}\left(\overline{K \cup T_{b}(K)}\right) .
$$


We now need to impose mixed boundary conditions on $\Omega_{1}$ and $\Omega_{2}$. To do so, let us first decompose $\partial_{0} K$ into the union of two non-intersecting sets $\partial_{0, D} K$ and $\partial_{0, N} K$ (one of which may be empty). Then we set (see figure 2)

$$
\begin{aligned}
& \partial_{D} \Omega_{1}:=\partial_{0, D} K \cup T_{a}\left(\partial_{0, D} K\right) \cup T_{a}\left(\partial_{b} K\right) ; \\
& \partial_{N} \Omega_{1}:=\partial_{0, N} K \cup T_{a}\left(\partial_{0, N} K\right) \cup \partial_{b} K ; \\
& \partial_{D} \Omega_{2}:=\partial_{0, D} K \cup T_{b}\left(\partial_{0, D} K\right) \cup \partial_{a} K ; \\
& \partial_{N} \Omega_{2}:=\partial_{0, N} K \cup T_{b}\left(\partial_{0, N} K\right) \cup T_{b}\left(\partial_{a} K\right) ;
\end{aligned}
$$

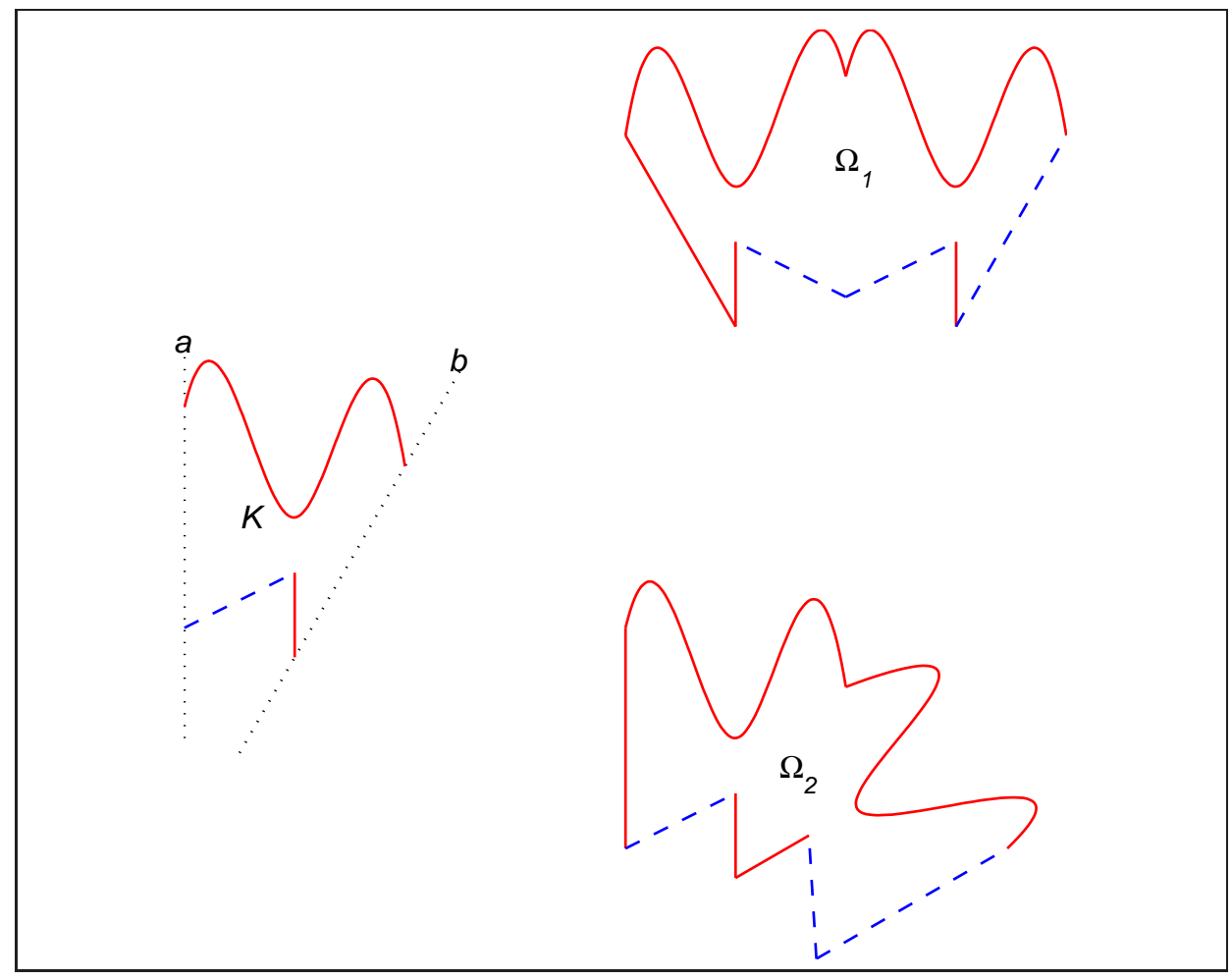

Figure 2. A generic construction block and resulting domains $\Omega_{1}$ and $\Omega_{2}$.

Theorem 3.1. For any choice of lines $a, b$, of a "construction block" $K$, and of its boundary decomposition $\partial_{0, D} K$ and $\partial_{0, N} K$, we have

$$
\sigma_{D N}\left(\Omega_{1}\right)=\sigma_{D N}\left(\Omega_{2}\right)
$$

with the account of multiplicities.

Proof. The theorem is proved using the transplantation technique developed in [Ber], Bus. We show that there is a one-to-one correspondence between the eigenfunctions on $\Omega_{1}$ and on $\Omega_{2}$. Let $u_{1}(x)$ be an eigenfunction of the mixed Dirichlet-Neumann boundary problem on $\Omega_{1}$. Let us represent $u_{1}(x)$ as follows:

$$
u_{1}(x)= \begin{cases}u_{11}(x), & x \in K, \\ u_{12}\left(T_{a} x\right), & x \in T_{a} K,\end{cases}
$$


where $u_{11}(x), u_{12}(x)$ are functions on $K$ satisfying

$$
u_{11}(x)=u_{12}(x), \partial_{n} u_{11}(x)=-\partial_{n} u_{12}(x),
$$

for $x \in \partial_{a} K$. Let

$$
u_{21}(x)=u_{11}(x)-u_{12}(x), \quad u_{22}(x)=u_{11}(x)+u_{12}(x) .
$$

One can check by inspection that the function

$$
u_{2}(x)= \begin{cases}u_{21}(x), & x \in K, \\ u_{22}\left(T_{b} x\right), & x \in T_{b} K,\end{cases}
$$

is an eigenfunction of the corresponding mixed Dirichlet-Neumann boundary value problem on $\Omega_{2}$. It is easy to see that inverting this procedure one obtains an eingenfunction of the problem on $\Omega_{1}$ from an eigenfuntion of the corresponding problem on $\Omega_{2}$. Note also that since (3.3) is a linear transformation we get $\sigma_{D N}\left(\Omega_{1}\right)=\sigma_{D N}\left(\Omega_{2}\right)$ with the account of multiplicities. This completes the proof of the theorem.

The construction of this section also gives us the basic example of section 2 if we take the "construction block" $K$ to be an isosceles right-angled triangle with legsize one, $a$ being the hypotenuse of $K, b$ being one of the legs, and $\partial_{0, N} K$ chosen to be empty.

\section{Isospectrality and multi-sheeted coverings of $K$}

In this section, we indicate an alternative way of proving Theorem 3.1, and, at the same time, relate in an indirect way the spectra $\sigma_{D N}\left(\Omega_{j}\right)$ and the spectra of boundary value problems on the "construction block" $K$. For illustrative purposes all the figures in this section use a construction block $K$ with parallel sides $a, b$, which is different from the construction block shown in the previous section.

To start with, consider an eight-sheet covering $\stackrel{\curvearrowright}{K}_{8}$ of the block $K$. It is constructed by "gluing" together four copies of $K$ and four copies of its reflection $T_{a} K$, and identifying the outer edges, as shown in Figure 3 .

The dotted lines show the original bounding lines and their images under reflections; we set

$$
a_{0}:=b, \quad a_{1}:=a, \quad a_{j}:=T_{a_{j-1}}\left(a_{j-2}\right) \text { for } j=2, \ldots, 8
$$

and identify $a_{0}$ and $a_{8}$.

$\overbrace{K_{8}}$ is a flat manifold with boundary on which we consider a mixed DirichletNeumann problem with boundary conditions imposed according to the original choice of $\partial_{0, D} K$ and $\partial_{0, N} K$ and their reflections. Denote the spectrum of the corresponding Laplacian by $\sigma\left(\stackrel{\curvearrowright}{\kappa_{8}}\right)$.

Any pair of lines $a_{k} \cup a_{k+4}, k=0, \ldots, 4$ defines a symmetry on $\stackrel{\curvearrowright}{K}_{8}$ which preserves both $a_{k}$ and $a_{k+4}$ and exchanges $a_{(k-j) \bmod 8}$ with $a_{(k+j) \bmod 8}$ for $j=1,2,3$. Consider, e.g., the case $k=0$. Then the lines $a_{0}, a_{4}$ split $\stackrel{\curvearrowright}{K}_{8}$ into two identical domains; denote 


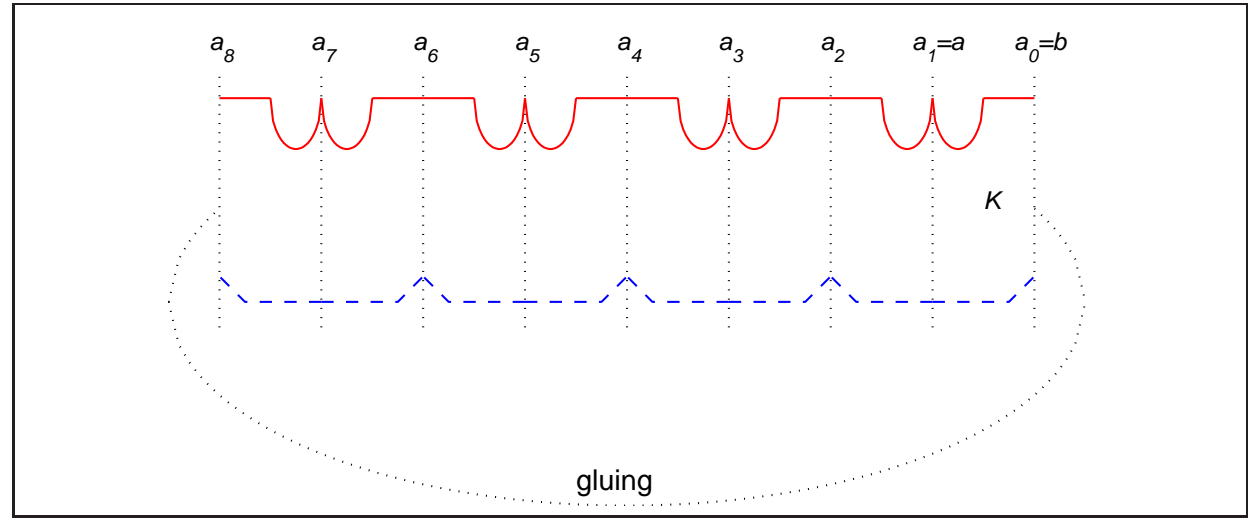

Figure 3. $\stackrel{\curvearrowright}{K}_{8}$, an eigth-sheeted covering of $K$. Here the construction block $K$ is bounded by two parallel lines $a$ and $b$. The main construction of section 3 gives an isospectral pair, with $\Omega_{1}$ being bounded by $a_{2}$ (with the Dirichlet condition imposed) and $a_{0}$ (Neumann) and $\Omega_{2}$ being bounded by $a_{3}$ (Dirichlet) and $a_{1}$ (Neumann).

one of them $K_{4}$. The eigenfunctions on $\stackrel{\curvearrowright}{K_{8}}$ can be chosen in such a way that each one shall satisfy either a Dirichlet or a Neumann boundary condition on $a_{0} \cup a_{4}$, and

$$
\sigma(\overbrace{\bar{K}})=\sigma_{D D}\left(K_{4}\right) \cup \sigma_{N N}\left(K_{4}\right)
$$

where $\sigma_{D D, N N}\left(K_{4}\right)$ stand for the spectra of the Laplacian on $K_{4}$ with corresponding boundary conditions imposed on $a_{0}$ and $a_{4}$, and the union is understood with account of multiplicities.

Consider now these two new problems on $K_{4}$. For each of them, $a_{2}$ is the line of symmetry which divides $K_{4}$ into two copies of $\Omega_{1}$. By the same argument,

$$
\sigma_{D D}\left(K_{4}\right)=\sigma_{D N}\left(\Omega_{1}\right) \cup \sigma_{D D}\left(\Omega_{1}\right) \quad \text { and } \quad \sigma_{N N}\left(K_{4}\right)=\sigma_{N D}\left(\Omega_{1}\right) \cup \sigma_{N N}\left(\Omega_{1}\right) ;
$$

here again the indices $D$ and $N$ correspond to the boundary conditions imposed on the sides $a_{0}$ and $a_{2}$ of $\Omega_{1}$. Obviously, $\sigma_{N D}\left(\Omega_{1}\right)=\sigma_{D N}\left(\Omega_{1}\right)$ by symmetry.

Repeating the argument once more for symmetric problems on $\Omega_{1}$, we get

$$
\sigma_{D D}\left(\Omega_{1}\right)=\sigma_{D N}(K) \cup \sigma_{D D}(K) \quad \text { and } \quad \sigma_{N N}\left(\Omega_{1}\right)=\sigma_{N D}(K) \cup \sigma_{N N}(K),
$$

and so

$$
\sigma_{D N}\left(\Omega_{1}\right) \cup \sigma_{D N}\left(\Omega_{1}\right)=\sigma\left(\stackrel{\curvearrowright}{K}_{8}\right) \backslash\left(\sigma_{D D}(K) \cup \sigma_{D N}(K) \cup \sigma_{N D}(K) \cup \sigma_{N N}(K)\right),
$$

i.e. the spectrum $\sigma_{D N}\left(\Omega_{1}\right)$ taken with double multiplicities is obtained by removing from the spectrum $\sigma(\overbrace{\bar{K}})$ of the problem on the eight-sheeted covering the spectra of the four boundary value problems on the "construction block" $K$.

Let us repeat now the whole process but start with considering the symmetry with respect to $a_{1} \cup a_{5}$. Then instead of $K_{4}$ we should consider a different set $K_{4}^{\prime}$ bounded by $a_{1}$ and $a_{5}$, which has a line of symmetry $a_{3}$ dividing it into two copies of $\Omega_{2}$. The previous argument gives again (4.1), with $\Omega_{1}$ replaced by $\Omega_{2}$, thus providing an alternative proof of Theorem 3.1 . 
Further on, a similar argument provides us with another pair of isospectral domains with more complicated symmetries than those of the previous section. Namely, we have Theorem 4.2.

$$
\sigma_{D N}\left(K_{4}\right)=\sigma_{D N}\left(K_{4}^{\prime}\right),
$$

or more generally

$$
\sigma_{D N}\left(K_{2^{n}}\right)=\sigma_{D N}\left(K_{2^{n}}^{\prime}\right)
$$

where $K_{2^{n}}$ is constructed by gluing together $2^{n-1}$ copies of $K$ and $2^{n-1}$ copies of $T_{a} K$ starting with $K$, and $K_{2^{n}}^{\prime}$ is constructed by gluing together $2^{n-1}$ copies of $K$ and $2^{n-1}$ copies of $T_{b} K$ starting with $K$.

See Figure 4 for illustration in the case $n=2$. The proof is by induction in $n$ using the argument similar to the one above via the construction of $2^{n+3}$-multiple covering $\overbrace{K_{2}}^{n+3}$. We omit the details of the argument.

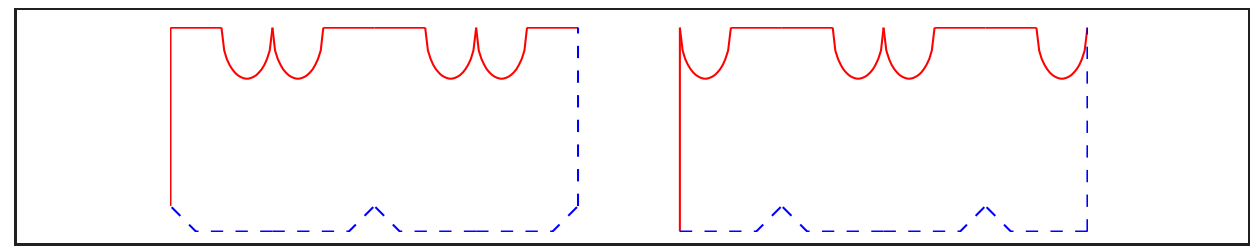

Figure 4. Isospectral problems on $K_{4}$ and $K_{4}^{\prime}$. The construction block $K$ is the same as in Figure 3

\section{Necessary conditions for mixed isospectrality}

We present here some necessary conditions for mixed isospectrality coming from the heat trace asymptotic expansion for a mixed Dirichlet-Neumann boundary problem.

Theorem 5.1. Let two domains $\Omega_{j}, j=1,2$, with boundary decompositions $\partial \Omega_{j}=$ $\overline{\partial_{D} \Omega_{j} \cup \partial_{N} \Omega_{j}}$, be isospectral in the above sense. Then the following quantities should coincide for $j=1$ and $j=2$ :

- $\operatorname{Area}\left(\Omega_{j}\right)$;

- Length $\left(\partial_{D} \Omega_{j}\right)-\operatorname{Length}\left(\partial_{N} \Omega_{j}\right)$;

- $2 \int_{\partial \Omega_{j}} \kappa(s) \mathrm{d} s+\sum_{D D} \frac{\pi^{2}-\beta^{2}}{\beta}+\sum_{N N} \frac{\pi^{2}-\beta^{2}}{\beta}-\frac{1}{2} \sum_{D N} \frac{\pi^{2}+2 \beta^{2}}{\beta}$.

In the last formula, $\kappa$ is the curvature of the boundary, and the sums are taken over all corners of $\partial \Omega_{j}$ formed by Dirichlet-Dirichlet (DD), Neumann-Neumann (NN), and Dirichlet-Neumann (DN) parts of the boundary, respectively; in each case $\beta$ is a corresponding angle.

Proof. The theorem follows immediately from the formulae for the first three heat trace coefficients in the case of a mixed Dirichlet-Neumann problem (see [Dow]) - as the spectra coincide, the heat trace expansions should coincide as well. 


\section{More examples}

In this section, we illustrate our construction by more examples. They are quite simple and do not require explanation apart from graphical one. In all the cases we show the construction block and resulting isospectral domains.

Example 1. Simply connected and not simply connected domains. As in Figure 5.

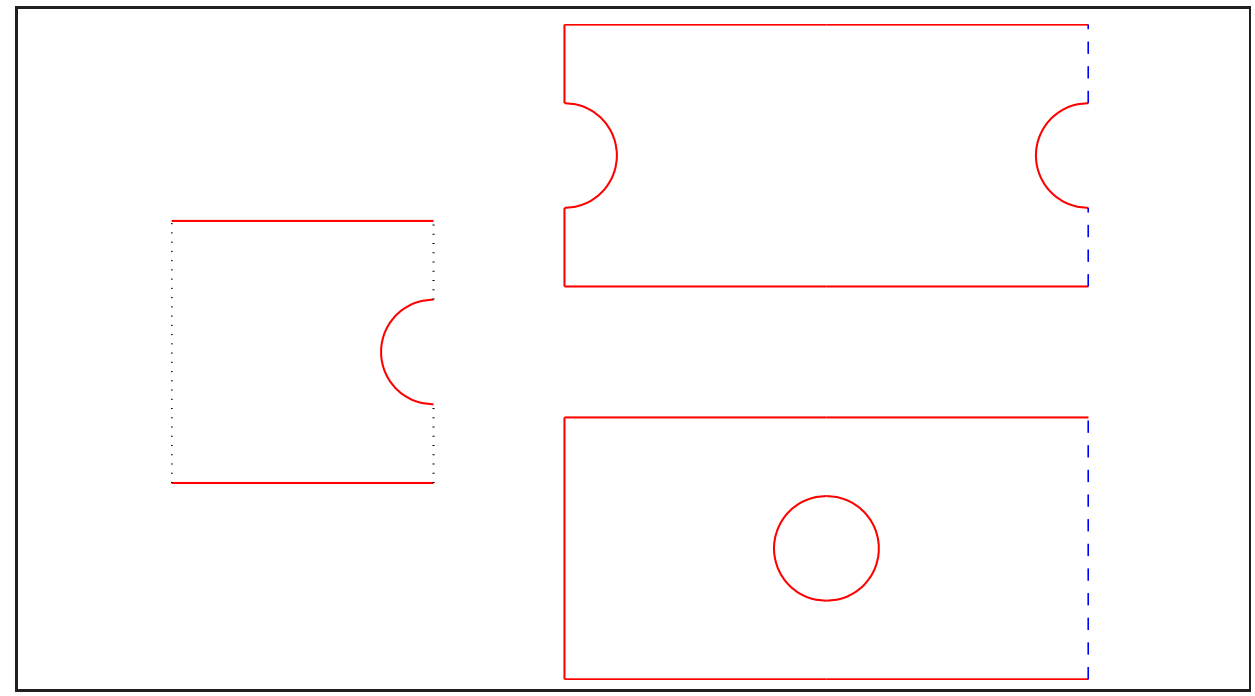

Figure 5. Isospectral simply connected and non-simply connected domains.

Example 2. One smooth and one non-smooth domain. As in Figure 6. Note that the boundary of the upper domain is smooth but not analytic.

Example 3. Four isospectral domains. This example is more complicated than the two previous ones.

We start with a construction block lying inside a rectangle formed by two pairs of parallel straight lines, $a, b$, and $c$, $d$, see Figure 7 . We reflect first with respect to the line $a$ and impose the Dirichlet (resp., Neumann) condition on $T_{a} b$ (resp., $b$ ) thus giving us the domain on the left of the middle row of Figure 7 . We now reflect it with respect to the lines $c$ or $d$ and impose different boundary conditions on opposite straight lines, thus giving us (by the argument of section 3) a pair of isospectral domains $\Omega_{1}$ and $\Omega_{2}$.

If we reflect first with respect to the line $b$ and impose the Dirichlet (resp., Neumann) condition on $a$ (resp., $T_{b} a$ ) we obtain the domain shown on the right of the middle row of Figure [7. Again reflecting with respect to $c$ and $d$, we obtain another isospectral pair $\Omega_{3}$ and $\Omega_{4}$.

It remains therefore to show that both pairs are isospectral to each other. To do this we notice that reflecting first with respect to $c$, and then with respect to $a$ and 


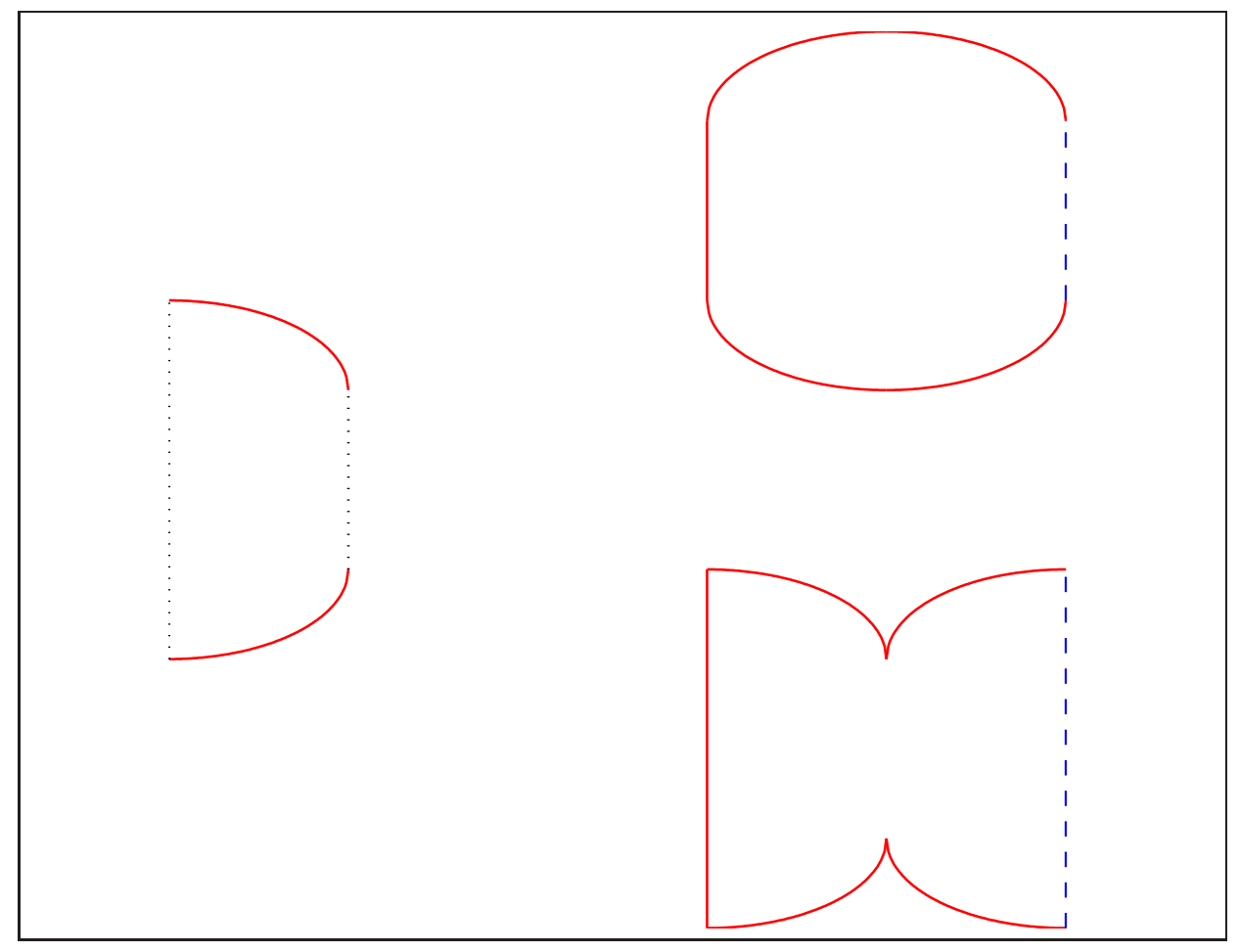

Figure 6. Isospectral smooth and non-smooth domains.

$b$ proves, by the same argument, the isospectrality of the resulting pair $\Omega_{1}$ and $\Omega_{3}$. Therefore, all four domains are isospectral. Note also that $\Omega_{2}$ is non-simply connected and the other domains are simply connected, thus extending Example 1.

More complicated constructions producing sets of $2^{n}$ isospectral non-isometric domains are possible in any dimension $n \geq 2$.

Example 4. Domains isospectral with respect to Dirichlet-Neumann swap. This is discussed at length in [JLNP, where we construct a series of examples of boundary value problems whose spectrum remains the same when swapping Dirichlet and Neumann parts of the boundary. The main example is of two problems on a halfdisk, see Figure 8

This example has an unexpected application [JLNNP, which has in fact first attracted our attention to Dirichlet-Neumann isospectrality.

\section{Can one count the shape of a drum?}

Very recently, Uzy Smilansky and collaborators have noticed GnSmSo, ShSm that in some situations pairs of previously known isospectral objects (manifolds or graphs) can be distinguished by comparing their nodal sequences. Namely, if $\lambda_{m}$ is the $m$-th eigenvalue and $u_{m}$ is a corresponding eigenfunction of the Dirichlet Laplacian on $\Omega$, denote by $N_{m}(\Omega):=\left\{x \in \Omega: u_{m}(x)=0\right\}$ the nodal set of $u_{m}$ and by $\nu_{m}(\Omega)$ the number 


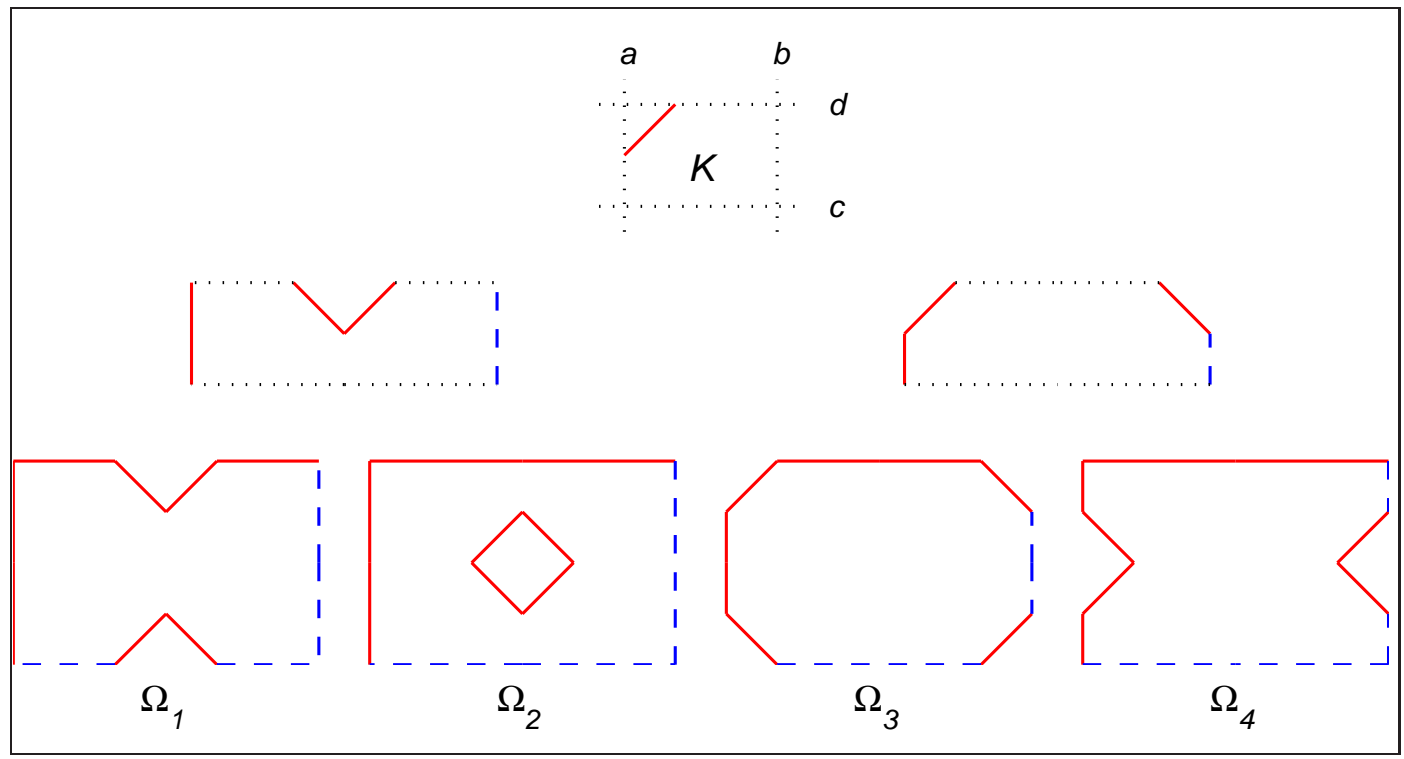

Figure 7. Four isospectral domains.

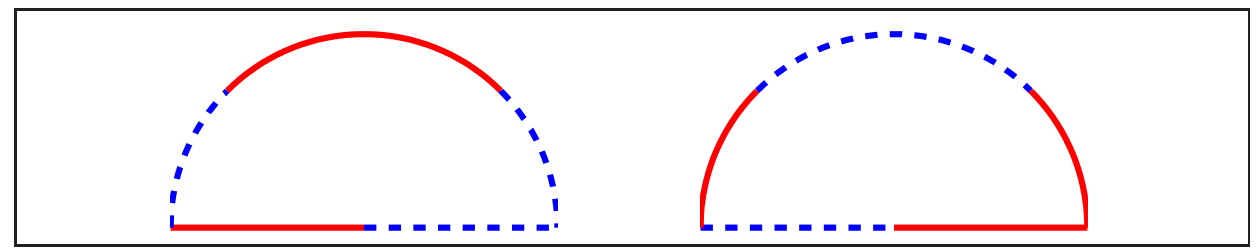

Figure 8. Two problems on a half-disk.

of connected components of $\Omega \backslash N_{m}(\Omega)$. Note that $\nu_{m}(\Omega)$ is only well defined in such a way for a simple eigenvalue $\lambda_{m}$ and that the case of multiple eigenvalues requires some special treatment.

Numerical experiments in GnSmSo, ShSm based on statistical analysis of a normalized nodal domain count, see BlGnSm, showed that isospectral pairs provide in fact different distributions of nodal domains.

We note here that, at least for some low eigenvalues, the pairs of isospectral domains with mixed boundary conditions considered in this paper also produce different nodal count. Consider, e.g. our basic example of a square and a triangle in section 2. The nodal domains for the eigenfunctions corresponding to the fourth eigenvalues $\lambda_{1,2}=\mu_{3,0}$ are shown in Figure 9, and we see that $\nu_{4}\left(\Omega_{1}\right)=4 \neq 3=\nu_{4}\left(\Omega_{2}\right)$.

As finite element calculations show, the nodal domain count is also different, e.g., for the third eigenfunctions of the two problems in the half-disk isospectral with respect to the Dirichlet-Neumann swap (Example 4 of the previous section) with four and two nodal domains, respectively.

It would be of course interesting to prove that any two isospectral mixed DirichletNeumann problems can be distinguished by their nodal count; this question remains open. 


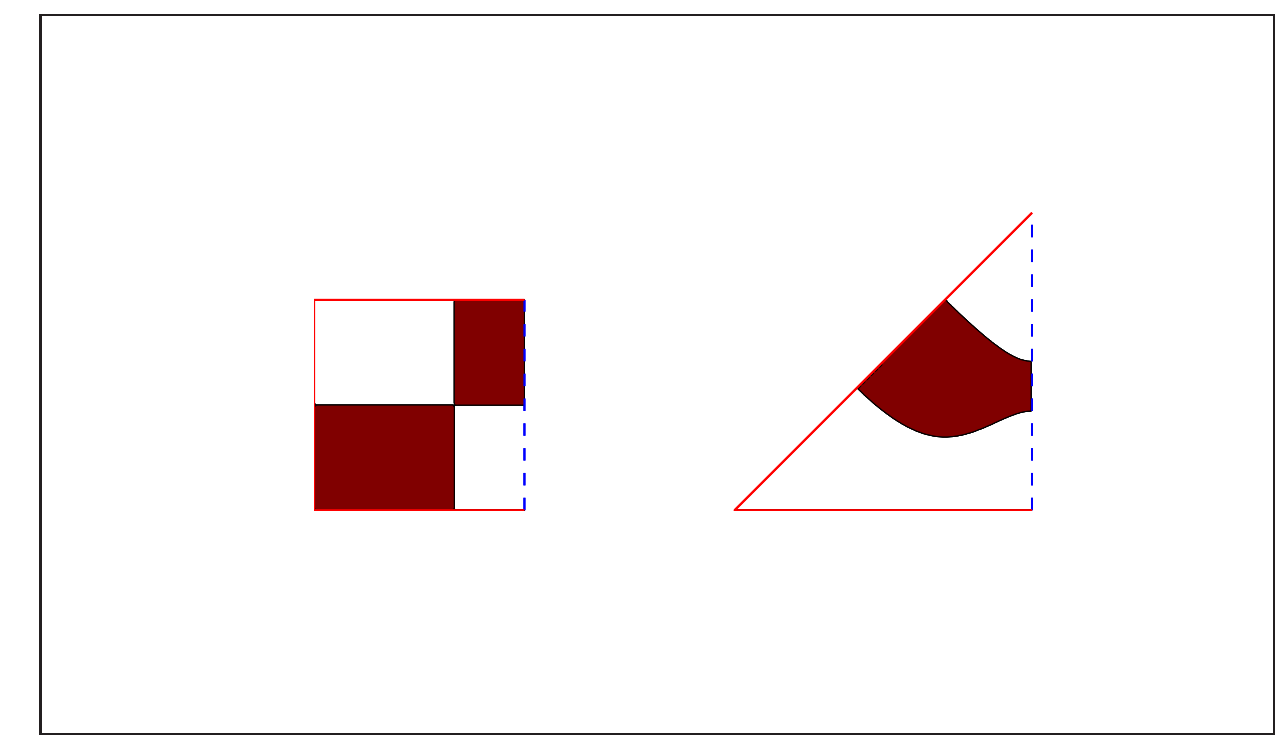

Figure 9. Nodal domains for the basic example of section 2.

\section{Acknowledgments}

The authors are grateful to Uzy Smilansky for valuable discussions. The research of I.P. was partially supported by NSERC and FQRNT.

\section{References}

[Ber] Berard P 1992 Transplantation et isospectralité I Math. Ann. 292 547-559.

[BetTr] Betcke T and Trefethen L N 2005 Reviving the method of particular solutions SIAM Review $47469-491$.

[BlGnSm] Blum G, Gnutzmann S and Smilansky U 2002 Nodal domains statistics: a criterion for quantum chaos Phys. Rev. Lett. 88 114101-1-114101-4.

[Bro] Brooks R 1988 Constructing isospectral manifolds Amer. Math. Monthly 95 823-839.

[Bus] Buser P 1986 Isospectral Riemannian surfaces Ann. Inst. Fourier 36 167-192.

[BCDS] Buser P, Conway J, Doyle P and Semmler K-D 1994 Some planar isospectral domains Int. Math. Res. Notes 9 391-400.

[Ch] Chapman S J (1995) Drums that sound the same Amer. Math. Monthly 102 124-138.

[DG] Driscoll T and Gottlieb H P W 2003 Isospectral shapes with Neumann and alternating boundary conditions Phys. Rev. E 68 016702-1 - 016702-6.

[Dow] Dowker J S 2005 The hybrid spectral problem and Robin boundary conditions J. Phys. A: Math. Gen. 38 4735-4754.

[GnSmSo] Gnutzmann S, Smilansky U and Sondergaard N 2005 Resolving isospectral 'drums' by counting nodal domains J. Phys. A: Math. Gen. 38 8921-8933.

[GWW] Gordon C, Webb D and Wolpert S 1992 Isospectral plane domains and surfaces via Riemannian orbifolds Invent. Math. 110 1-22.

[JLNNP] Jakobson D, Levitin M, Nadirashvili N, Nigam N and Polterovich I 2005 How large can the first eigenvalue be on a surface of genus two? Int. Math. Res. Notices 63 3967-85.

[JLNP] Jakobson D, Levitin M, Nadirashvili N and Polterovich I 2004 Spectral problems with mixed Dirichlet-Neumann boundary conditions isospectrality and beyond Preprint math.SP/0409154 (to appear in J. Comp. Appl. Math. doi:10.1016/j.cam.2005.06.019). 
[Kac] Kac M 1966 Can one hear the shape of a drum? Amer. Math. Monthly 73 1-23.

[ShSm] Shapira T and Smilansky U 2005 Quantum graphs which sound the same in preparation.

[SlHu] Sleeman B D and Hua C 2000 On nonisometric isospectral connected fractal domains Rev. Mat. Iberoamericana 16 351-361.

[Sun] Sunada T 1985 Riemannian coverings and isospectral manifolds Ann. Math. 121 169-186.

[Za] Zaremba S 1927 Sur un problème toujours possible comprenant à titre de cas particuliers, le problìne de Dirichlet et celui de Neumann J. Math. Pure Appl. 6 127-163. 